\title{
The Intriguing New Cataclysmic Variable KUV 03580+0614
}

\author{
Paula Szkody円 \\ Department of Astronomy, University of Washington, Seattle, WA 98195 \\ szkody@astro.washington.edu \\ Boris Gänsicke \\ Universitäts-Sternwarte Göttingen, Geismarlandstr. 11, 37073 Göttingen, Germany \\ boris@uni-sw.gwdg.de \\ Robert E. Fried \\ Braeside Observatory, PO Box 906, Flagstaff AZ 86002, captain@braeside.org \\ Uli Heber \\ Sternwarte Bamberg, ai03@sternwarte.uni-erlangen.de \\ Dawn K. Erb \\ Department of Astronomy, CalTech, Pasadena, CA, dke@astro.caltech.edu
}

\begin{abstract}
Photometric and spectroscopic observations of the candidate cataclysmic variable KUV 03580+0614, independently found from the Hamburg survey as HS $0357+0614$, reveal this to be a cataclysmic variable with an orbital period of 3.4 hrs. CVs with periods in this range are usually novalikes belonging to the SW Sex or Intermediate Polar categories. The variability, spectroscopic characteristics and the lack of strong X-rays indicate a system similar to V795 Her, LS Peg and AH Men.
\end{abstract}

Subject headings: cataclysmic variables — stars:individual (KUV 03580+0614)

— spectroscopy:stars — photometry:stars

\footnotetext{
${ }^{1}$ Based on observations with the Apache Point Observatory (APO) 3.5m telescope, which is owned and operated by the Astrophysical Research Consortium (ARC)
} 


\section{Introduction}

The Hamburg Schmidt objective prism survey (HQS; Hagen et al. 1995) provides a magnitude-limited $(V=13-17.5)$ sample of low resolution spectra $\left(\sim 45 \AA\right.$ at $\left.\mathrm{H}_{\gamma}\right)$ of blue objects. Although it was designed to pick up quasars, it has proven to be a resource for finding blue stars (see e.g. Heber, Jordan \& Weidemann 1991) including new cataclysmic variables $(\mathrm{CVs})$. Recent discoveries include the UX UMa type HS 0139+0559 (Heber, Jordan \& Weidemann 1991), the eclipsing systems HS 1804+6753 (EX Dra; Billington et al. 1996) and HS 0907+1902 (Gänsicke et al. 2000) and the magnetic systems HS 1023+3900 (Reimers, Hagen \& Hopp 1999) and HS 0922+1333 (Reimers \& Hagen 2000). We have independently identified the $15.5 \mathrm{mag}$ CV candidate KUV 03580+0614 from the Kiso Schmidt camera survey (Wegner \& Boley 1993) as HS 0357+0614 during the course of follow-up studies of hot star candidates from the HQS. Our preliminary low resolution spectrum showed prominent HeII $\lambda 4686$ emission, as is also evident in the Wegner \& Boley spectrum. This line is a characteristic signature of either a magnetic CV (AM Her or Intermediate Polar type) or an old nova (the various types of CV systems and their characteristics are reviewed in Warner 1995). In order to determine the specific nature of KUV 03580+0614, we obtained photometric and time-resolved spectroscopic observations which provided the orbital period, the range of variability on short and long timescales and the location of the line emission regions.

\section{Observations}

Our identification spectrum of HS 0357+0614 (=KUV 03580+0614), obtained in October 1990 at the Calar Alto 3.5m telescope with the focal reductor spectrograph with a resolution of about $7 \AA$ (Figure 1 ), is characterized by Balmer and He I and He II emission as well as the $\mathrm{C}, \mathrm{N}$ blend at $4650 \AA$. The He II line is similar in strength to $\mathrm{H} \beta$, indicating the presence of a radiation field rich in high-energy photons. The overall appearance is similar to the lower resolution spectrum in Wegner \& Boley (1993).

Twenty higher resolution spectra over $3.5 \mathrm{hrs}$ were obtained at the Apache Point Observatory (APO) with the $3.5 \mathrm{~m}$ telescope and Double Imaging Spectrograph (DIS) on the night of 2000 January 11 UT. The DIS coverage is $4200-5050 \AA$ in the blue and $5800-6800 \AA$ in the red with $\sim 2.5 \AA$ resolution. A 1.5 arcsec slit was used with seeing conditions comparable to the slit width. The spectra were analyzed in a standard manner 
using IRAF.2 The images were corrected with bias and flat fields, the spectra were extracted to one dimension with sky subtraction, the wavelengths were calibrated using helium, neon and argon lamps, and the fluxes were calibrated from standard stars in the Kitt Peak standards atlas. The combined mean blue and red spectra are shown in Figure 1. The flux differences between the Calar Alto and APO spectra are likely due to the intrinsic variability of the system as described in Section 4.

Differential photometry in a $\mathrm{V}$ filter was obtained on 3 nights at Manastash Ridge Observatory (MRO) in 1999 Sept. and October using a TEK CCD on the $0.76 \mathrm{~m}$ telescope. The bulk of our differential photometry of KUV $03580+0614$ was obtained in December 1999 and January 2000 at the Braeside Observatory (BO) in Arizona, using a 0.4m reflector equipped with a SITe 512 CCD camera. $B, V$, and $R$ magnitudes of KUV $03580+0614$ were derived relative to the comparison stars C, C1, and C2 (Figure 2), all of which are included in the guide star catalog and in the USNO catalog. The last night was simultaneous with the APO spectra. The observations are summarized in Table 1.

\section{Photometry}

The $V$ light curves from MRO (Figure 3) and the $R$ light curves from BO (Figure 4) show a modulation of $\sim 3.5 \mathrm{~h}$, superimposed by flickering on time scales of $15-30 \mathrm{~min}$. The quasi-simultaneous $B, V$, and $I$ light curves (Figure 5) show that the modulation is only marginally color-dependent. From Figure 4, it is apparent that the amplitude of the modulation is quite variable, ranging from $0.2 \mathrm{mag}$ (Dec. 24) to $0.4 \mathrm{mag}$ (Dec. 17). The bottom plot (Jan. 11) shows that large changes are even possible from one orbit to the next.

To quantify this variation, we computed discrete fourier transforms (DFTs) from various subsets of our photometry. These consistently revealed a strong signal at $f \sim 7 \mathrm{~d}^{-1}$. Figure 6 shows the power spectrum obtained from the combination of all our data. The strongest signal is again found near $f=7 \mathrm{~d}^{-1}$ and shows a substructure of four possible periods. The uncertainty in cycle counts between our observation intervals prevents us from formally rejecting any of these possible periods. We folded all our photometric data with the four likely periods, and favor $P_{\text {orb }}=205.92 \mathrm{~min}$ as it produces the smoothest orbital light curve, even though $P_{\text {orb }}=205.57 \mathrm{~min}$ is slightly stronger in the power spectrum. A sine fit

\footnotetext{
${ }^{2}$ IRAF (Image Reduction and Analysis Facility) is distributed by the National Optical Astronomy Observatories, which are operated by AURA, Inc., under cooperative agreement with the national Science Foundation.
} 
to all the data gave a period of $205.80 \pm 0.02 \mathrm{~min}$, which is very close to our favored period.

In order to analyze the short-term fluctuations, we fit a sine with a fixed period, $P=P_{\text {orb }}=205.92 \mathrm{~min}$, to the data of each individual night, and pre-whitened the data with the best-fit sine curves. We then recomputed the fourier transform on the detrended data. A number of periods are present in the range $30-100 \mathrm{~d}^{-1}$ which have amplitudes consistent with the observed flickering. This intermittent presence of QPOs in KUV $03580+0614$ is very similar to the short-term variability of the novalike variable TT Ari (Tremko et al. 1996). In TT Ari, which has been considered for a long time as an intermediate polar candidate, these QPOs where interpreted either as the Kepler period at the inner edge of a magnetically truncated disc or as the beat between the magnetospheric period and the white dwarf spin period (e.g. Hollander \& van Paradijs 1992).

\section{Spectroscopy}

Throughout the 3.5 hrs of APO spectra, the emission lines are broad, with the Balmer lines showing double and single peak components while the He II line remains single-peaked. Some representative spectra are shown in Figure 7. We used centroid and Gaussian fitting routines within IRAF to measure the line velocities (Table 2). These velocities were then fit to a sinusoid to determine the period, semi-amplitude, and gamma velocities. The best fit with the minimum standard deviation occurred for the He II line and the resulting period (204 min) was consistent with that found from the longer photometric dataset (above). We then fixed the period at $206 \mathrm{~min}$ (as determined by the photometry) and found the best values for $\mathrm{K}$ and $\gamma$ by redoing the sine fits (Table 3). The final fit and the data for HeII is shown in Figure 8. After submitting our paper, we became aware of a preprint by Thorstensen and Taylor (2001), which used $\mathrm{H} \alpha$ data to determine a slightly longer period of $215 \mathrm{~min}$ (the cycle ambiguities in their data do not allow phasing together their 3 nights of observation nor any of their nights with ours). Fixing the sinusoid period at 215 min with our data produced the same values as shown in Table 3 (with a shift of 0.04 phase). The period of $215 \mathrm{~min}$ is the second strongest peak in our power spectrum (Figure 6). Although they are not common, there are systems known (e.g. TT Ari) that have shorter photometric periods - "negative superhumps" - than orbital periods (Warner 1995). However, rather than speculating further, we await more extensive spectroscopic and photometric data to determine if the $4 \%$ difference is real and persistent and merely regard our phases as uncertain by 0.04 .

In order to provide more information as to the nature of the accretion in this system, we computed doppler tomograms (Horne 1991). To construct these, we used a fourier-filtered 
back-projection program from Keith Horne that was modified for our computer and plotting needs by Donald Hoard. Since there is no eclipse to mark the zero phasing, we used the red-blue crossing of the emission line velocity from the HeII line which would correspond to inferior conjunction of the secondary if the emission lines originate from the white dwarf area. For our data, this phase 0 occurs at JD 2,451,554.650. The resulting tomograms for $\mathrm{H} \alpha, \mathrm{H} \beta$ and He II are shown in Figure 9. Each tomogram shows a completely different structure. The He II shows a spot at a location close to the white dwarf (the small offset from $\mathrm{V}_{x}=0$ is within the accuracy of our phase determination). The $\mathrm{H} \alpha$ line shows a concentration at 0 velocity with an outer ring, while the $\mathrm{H} \beta$ emission shows a half ring, with a lack of emission from phases 0.6-0.9. The $\mathrm{H} \alpha$ also shows less emission at these phases.

Since the simultaneous photometry from BO on this night (bottom plot in Figure 4) shows a large change in amplitude of the orbital modulation from the beginning to the end of the APO spectra, it is possible that the changing line intensities from variable mass transfer are causing some of the peculiarities in the tomograms. One of the basic assumptions of tomography is that the emitting regions are equally visible at all orbital phases so transient emission alters the correct picture. However, since the red and blue spectra are obtained simultaneously, this does not explain the large differences between the lines. The APO spectra start at phase 0.42 and end at 1.44 and the increased flux evident in the photometry and spectra begins about phase 1.2 so phases $0.6-0.9$ are in the low fluxes (and evident as low equivalent widths in the Balmer lines during these phases). However, phases 0.4-0.6 (where the equivalent widths are large and there is strong emission in the tomogram (near $\mathrm{V}_{x}=0, \mathrm{~V}_{y}=-300$ ) is also in this low flux interval. It will require further data obtained with less change within an orbit to sort out the stable configuration in this system. We can conclude that the high excitation line emission originates close to the white dwarf while the Balmer emission is partly from a disk structure.

From the simultaneous photometry/spectra on Jan. 11, we can determine the orbital

modulation peaks at phase $\sim 0.75$. This phase is near that where a hot spot, created by the interaction of the mass transfer stream with the disk, would typically be best viewed. If this area is optically thick, it may be a strong continuum source, but may not be a source of line emission.

\section{Conclusions}

While the orbital period of KUV 03580+0614 seems clearly determined to be near 3.4 hrs, the exact nature of this novalike system is ambiguous. The bulk of CVs that occupy periods between 3-4 hrs consist of both the high mass transfer novalike SW Sex stars and 
the magnetic IPs. In general, the distinguishing traits of the SW Sex stars are strong HeII, single-peaked emission lines and absorption appearing in the Balmer lines at some phases (usually near 0.5). They are generally low X-ray emitters. The IPs usually show a consistent spin-pulse and large hard X-ray flux, although some have QPOs and others have low X-ray flux. They can also have strong He II. KUV 03580+0614 has not been detected in the ROSAT All Sky Survey, so it is likely a faint X-ray source.

The presence of a photometric modulation that may be $4 \%$ shorter than the orbital period, the sporadic prescence of a flickering timescale near 20 min and large variability in the structure of the Balmer lines throughout the orbit make this system very similar to a handful objects in the 3-4 hr period range with unique properties such as AH Men (Buckley et al. 1993), V795 Her (Patterson \& Skillman 1994), LS Peg (Szkody et al. 1997) and TT Ari (Kraicheva et al. 1999). Whatever the affiliation of KUV 03580+0614, its presence in the 3-4 hrs period range provides another chance to study the bizarre behavior of this type of CV. These systems, characterized by rather high accretion rates, are flanked by the period gap for $P_{\text {orb }} \lesssim 3 \mathrm{hr}$ and by dwarf novae with much lower accretion rates, such as U Gem or IP Peg, for $P_{\text {orb }} \gtrsim 4$ hr. Besides the empirical determination of the accretion flow in these high $\dot{M}$ systems between $3-4 \mathrm{hrs}$, the underlying deficiency in our understanding of CV evolution is the cause of the high mass transfer rates - are they related to a peculiar characteristic of the primary or to more activity from the secondary?

We gratefully acknowledge Keith Horne and Donald Hoard for providing the programs for Doppler tomography.

BTG was supported by DLR/BMBF grant 50 OR 99036 . The HQS was supported by the Deutsche Forschungsgemeinschaft through grants Re 353/11 and Re 353/22. Braeside

Observatory acknowledges the support of The Research Corporation, The National Science Foundation (AST-92-180002), and the Fund of Astrophysical Research.

\section{REFERENCES}

Billington, I., Marsh, T. R., Dhillon, V. S. 1996, MNRAS, 278, 673

Buckley, D. A. H., Warner, B., Remillard, R. A., Tuohy, I. R., Sullivan, D. J. 1993. MNRAS, 265, 926

Gänsicke, B. et al. 2000, A\&A, 356, L79

Hagen, H. J., Groote, D., Engels, D., Reimers, D. 1995, A\&AS, 111, 195 
Heber, U., Jordan, S., Weidemann, V. 1991, in White Dwarfs, eds. G. Vauclair, E. Sion, Kluwer, NATO ASI 336, 109

Hellier, C. 1999, ASP Conf. Ser. 157, 1

Hollander, A., van Paradijs, J., 1992, A\&A, 265, 77

Horne, K. 1991, in Fundamental Properties of Cataclysmic Variable Stars, ed. A. Shafter (San Diego:SDSU), 23

Kraicheva, Z., Stanishev, V., Genkov, V., Iliev, C. 1999,A\&A, 351, 607

Patterson, J., Skillman, D. R. 1994, PASP, 106, 1141

Reimers, D., Hagen, H. J. 2000, A\&A, 358, L45

Reimers, D., Hagen, H. J., Hopp, U. 1999, A\&A, 343, 157

Szkody, P., Garnavich, P., Holberg, J., Silber, A., Pastwick, L. 1997, AJ, 113, 2276

Thorstensen, J. R., Taylor, C. J., 2001, MNRAS, in press (astro-ph/0105499)

Tremko, J. et al. 1996, A\&A, 312, 121

Warner, B. 1995, Cataclysmic Variable Stars, CUP

Wegner, G., Boley, F.I. 1993, AJ, 105, 660 
Fig. 1.- The 1990 Calar Alto identification spectrum (top) and the APO blue (middle) and red (bottom) mean of the 20 high resolution spectra obtained on 11 January 2000.

Fig. 2.- Finding chart $\left(8^{\prime} \times 8^{\prime}\right)$ for HS $0357+0614(=$ KUV 03580+0614) obtained from the Digitized Sky Survey. The coordinates of the star are $\alpha(2000)=04^{h} 00^{m} 37.1^{s}$ and $\delta(2000)=+06^{\circ} 22^{\prime} 46^{\prime \prime}$. Our three comparison stars are labelled C, C1, and C2.

Fig. 3.- Sample V light curves from MRO (plotted as differential magnitudes with respect to a comparison star) showing the $3.5 \mathrm{hr}$ modulation.

Fig. 4.- Sample R light curves from BO. The bottom plot is simultaneous with the APO spectra, which end at 1554.716 .

Fig. 5.- Quasi-simultaneous $B, V$, and $I$ light curves of KUV $03580+0614$.

Fig. 6.- Power spectrum of all the photometric data of KUV 03580+0614. The DFT of the window function (spectral window) is shown on top of the panel.

Fig. 7.- A sample of the APO spectra as a function of phase showing the structure in $\mathrm{H} \beta$ versus the single-peaked nature of HeII.

Fig. 8. - The best fit radial velocity curve to the He II data, with the fit parameters of Table 3. The two open circles may be contaminated by cosmic rays and were excluded from the fit.

Fig. 9.- The Doppler tomograms of a) He II, b) $\mathrm{H} \alpha$ and c) $\mathrm{H} \beta$. 
Table 1. Summary of Observations

\begin{tabular}{cccccl}
\hline \hline Date & UT Time & Obs & Data & Exp.(s) & Num. Obs \\
\hline 1990 Oct 04 & $01: 57-02: 37$ & CA & spectra & 900 & 1 \\
1999 Sep 23 & $08: 52-12: 59$ & MRO & V phot & 30 & 199 \\
1999 Oct 16 & $11: 37-12: 24$ & MRO & V phot & 30 & 83 \\
1999 Oct 17 & $10: 18-12: 47$ & MRO & V phot & 30 & 147 \\
1999 Dec 16 & $03: 04-10: 06$ & BO & R phot & 50 & 376 \\
1999 Dec 17 & $02: 31-10: 14$ & BO & R phot & 50 & 452 \\
1999 Dec 24 & $02: 22-09: 47$ & BO & R phot & 50 & 449 \\
1999 Dec 27 & $01: 23-09: 36$ & BO & B,V,I phot & $143,75,50$ & $96,94,99$ \\
2000 Jan 11 & $01: 54-08: 34$ & BO & R phot & 50 & 377 \\
2000 Jan 11 & $01: 35-05: 12$ & APO & spectra & 600 & 20 \\
\hline
\end{tabular}


Table 2. 2000 January 11 Velocities (km/s)

\begin{tabular}{rrrr}
\hline \hline Time (UT min) & He $\lambda 4686$ & $\mathrm{H} \alpha$ & $\mathrm{H} \beta$ \\
\hline 97.5 & -53 & 16 & 104 \\
105.0 & -115 & 0 & 63 \\
114.2 & -18 & -21 & -15 \\
125.5 & 3 & 26 & -3 \\
136.7 & 57 & 64 & 37 \\
148.0 & 29 & 68 & 3 \\
159.2 & 48 & 50 & -28 \\
170.5 & 41 & 86 & -177 \\
181.8 & 18 & 69 & -89 \\
193.0 & -14 & 8 & -197 \\
204.3 & 12 & 30 & -157 \\
215.5 & -2 & 51 & -72 \\
226.9 & -15 & 15 & -153 \\
238.2 & -46 & -21 & -186 \\
249.4 & -66 & -35 & -169 \\
260.7 & -138 & -57 & -219 \\
271.9 & -88 & -45 & -67 \\
283.7 & -96 & 116 & 120 \\
295.0 & -64 & 73 & 102 \\
306.4 & -38 & 68 & 81 \\
\hline & & &
\end{tabular}

Table 3. Radial Velocity Curves

\begin{tabular}{lcccc}
\hline \hline \multicolumn{1}{c}{ Line } & $\gamma$ & $\mathrm{K}$ & $\phi$ & $\sigma$ \\
\hline $\mathrm{H} \alpha$ & $22 \pm 1$ & $44 \pm 13$ & $-0.03 \pm 0.04$ & 31 \\
$\mathrm{H} \beta$ & $-59 \pm 2$ & $117 \pm 17$ & $-0.27 \pm 0.03$ & 50 \\
$\mathrm{He} 4686$ & $-18 \pm 0.3$ & $62 \pm 6$ & $0.00 \pm 0.01$ & 16 \\
\hline
\end{tabular}



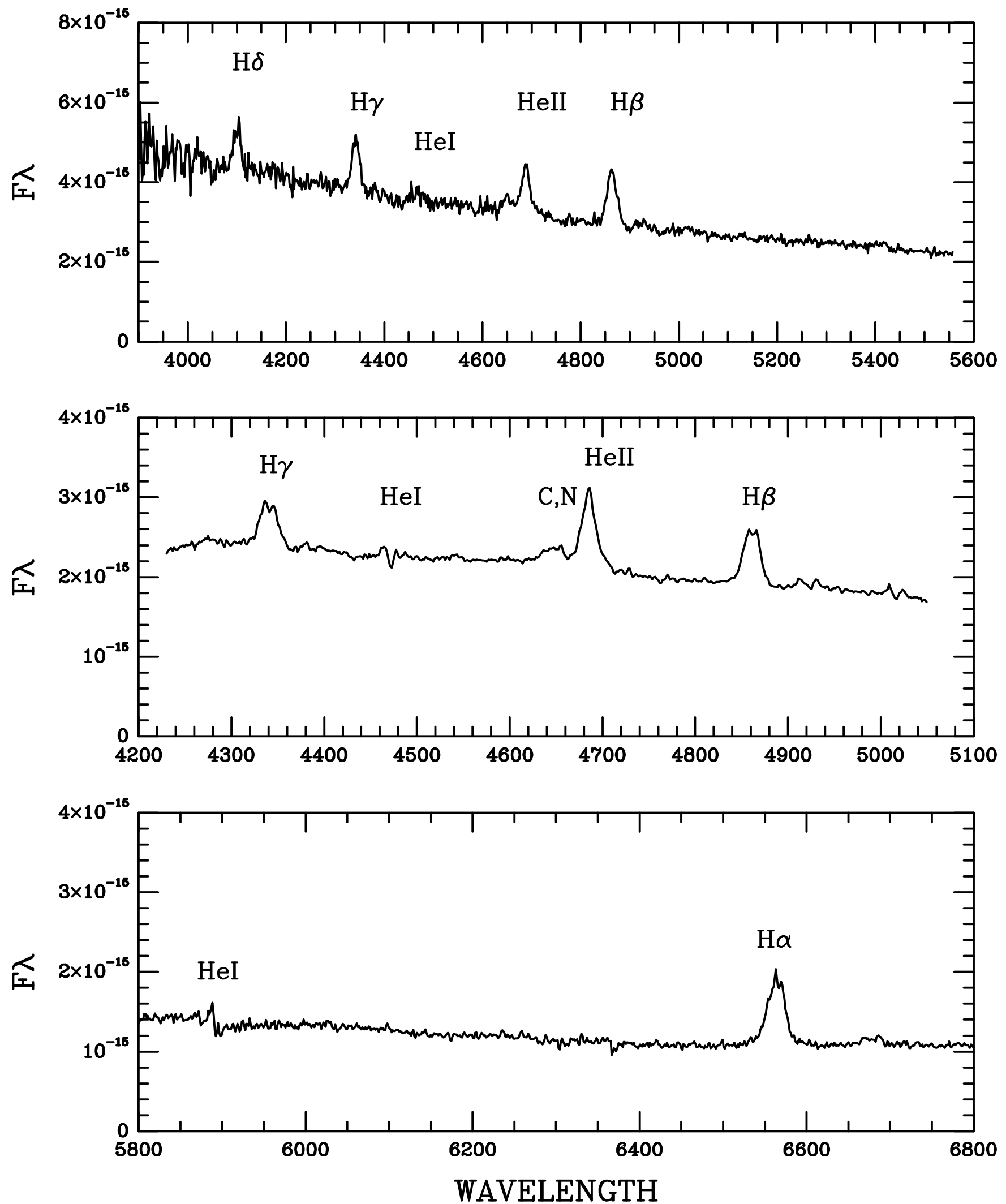


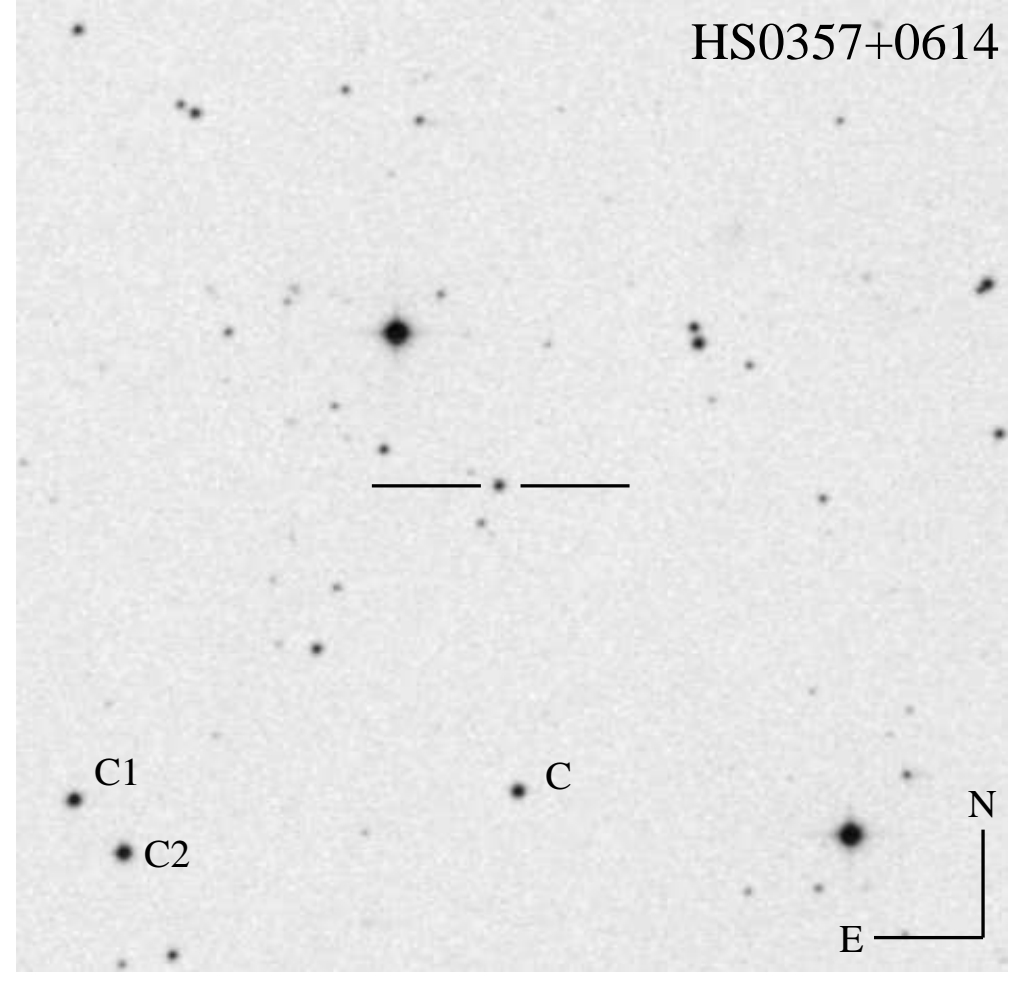



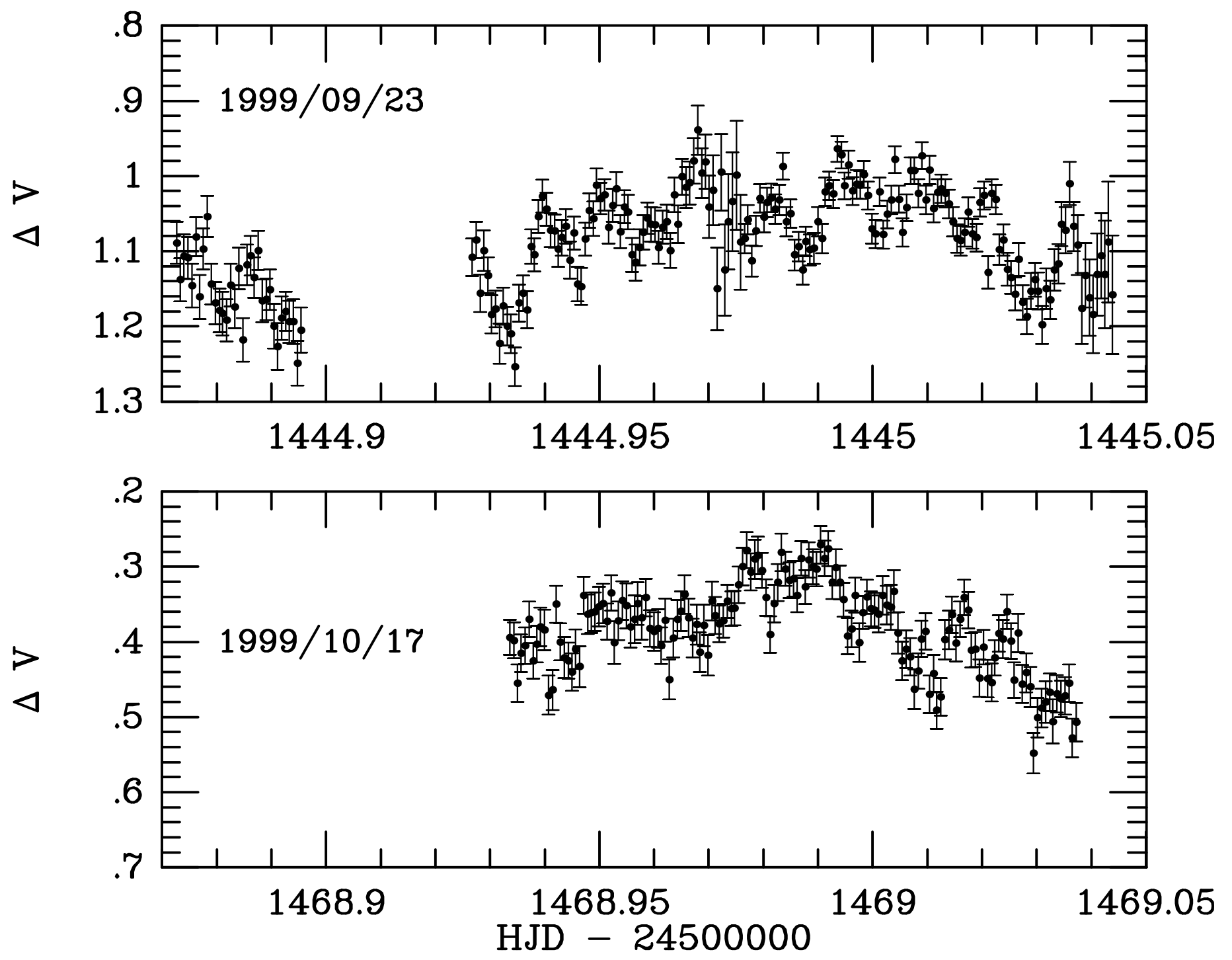

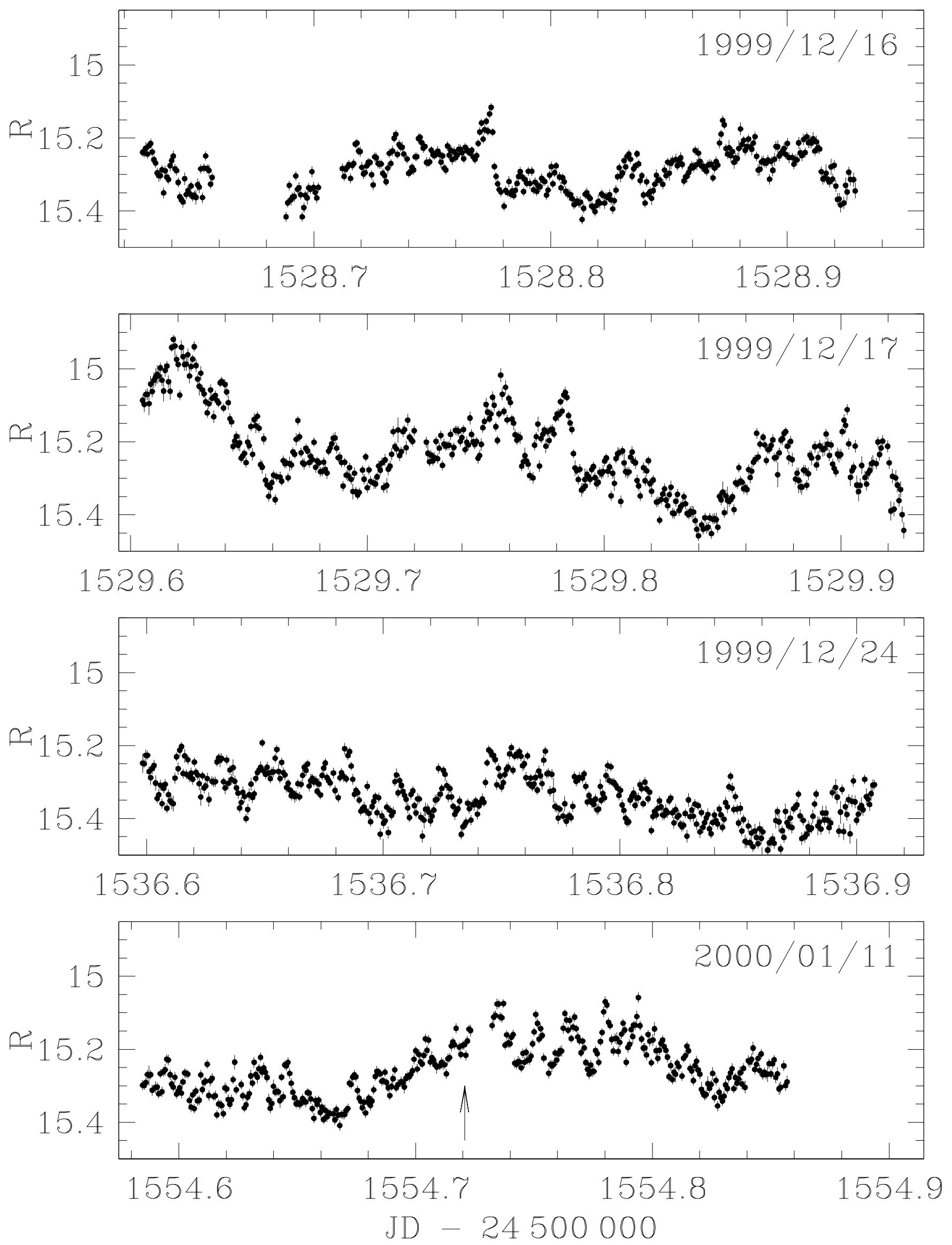


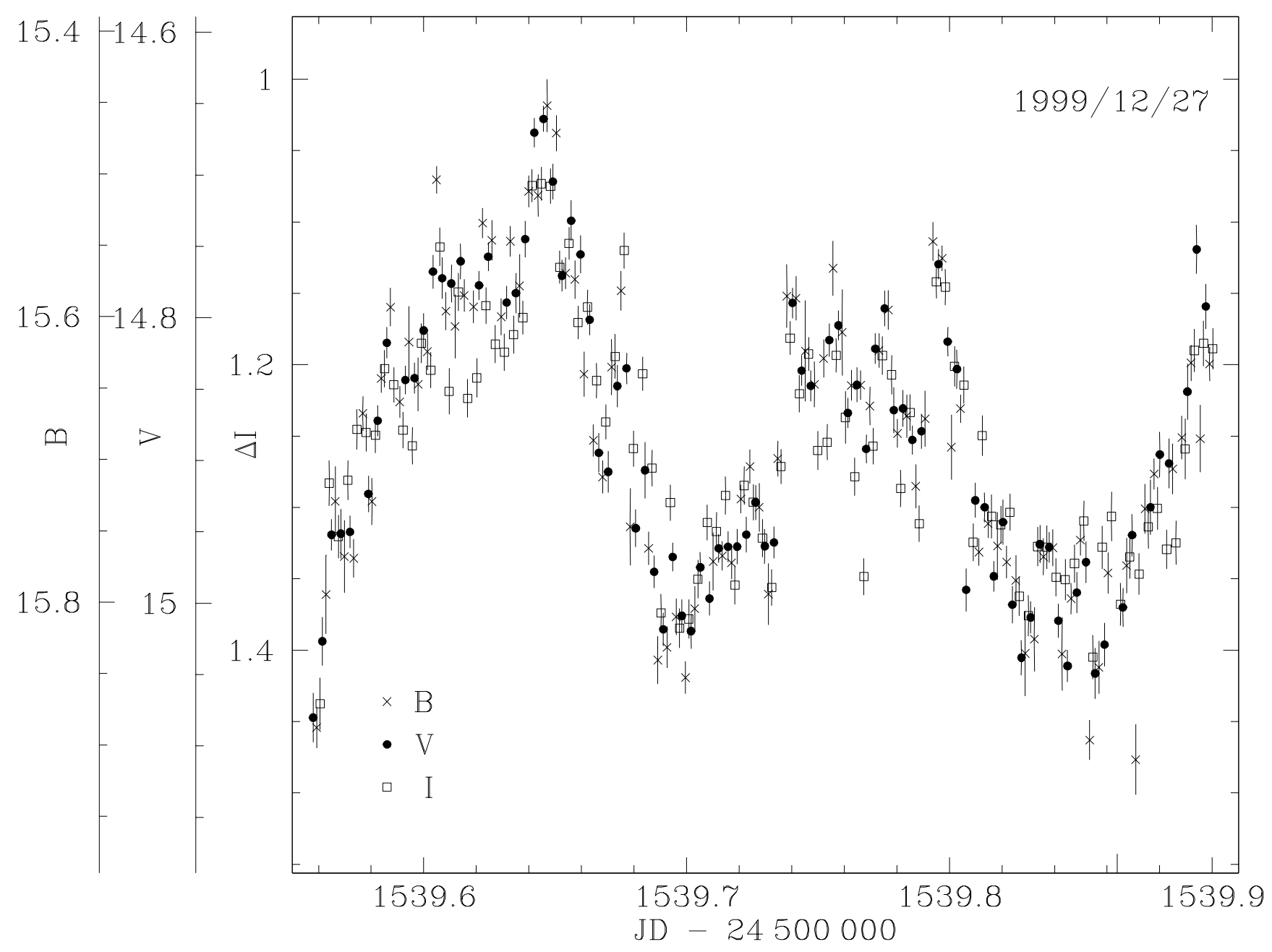




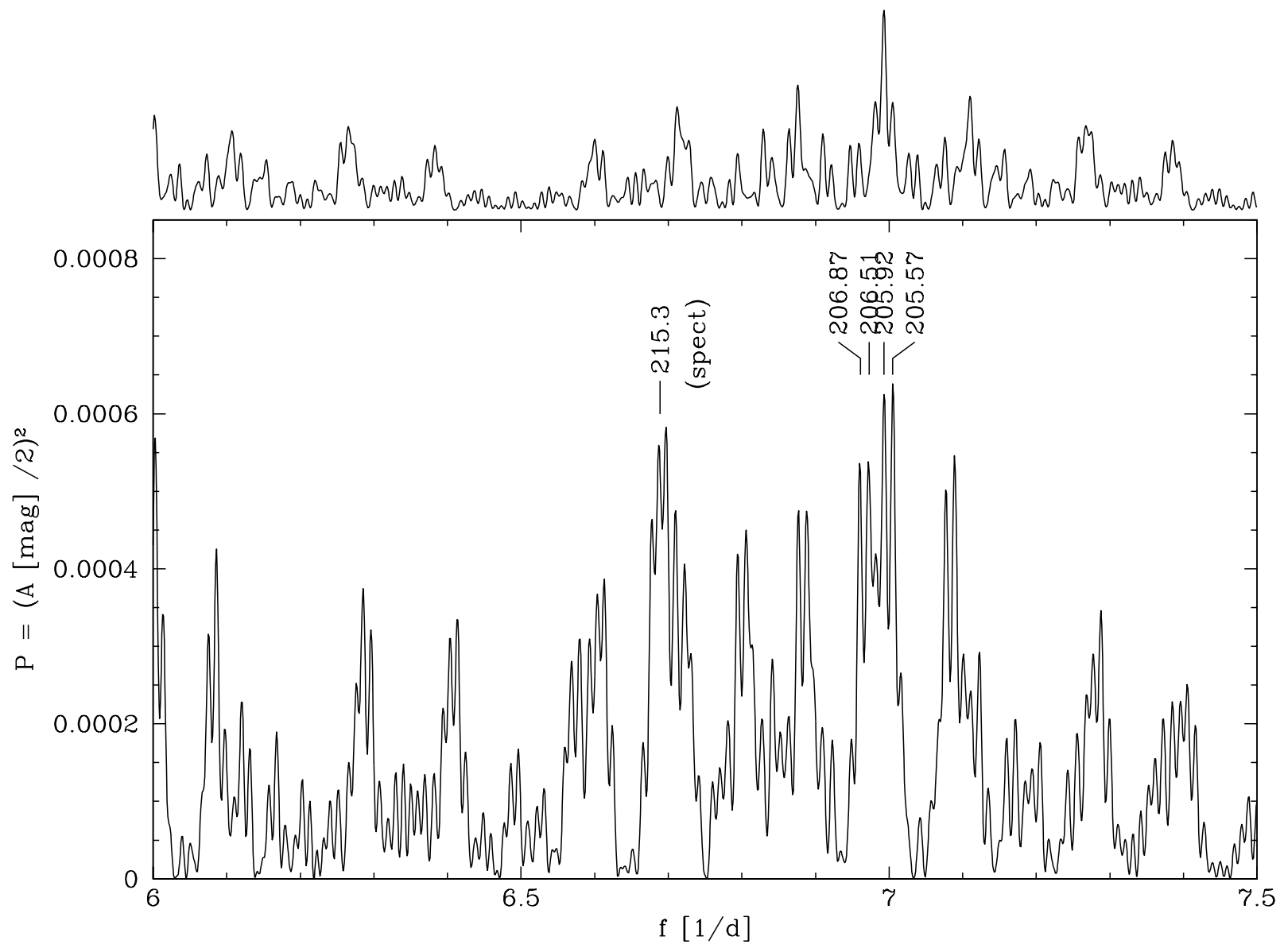




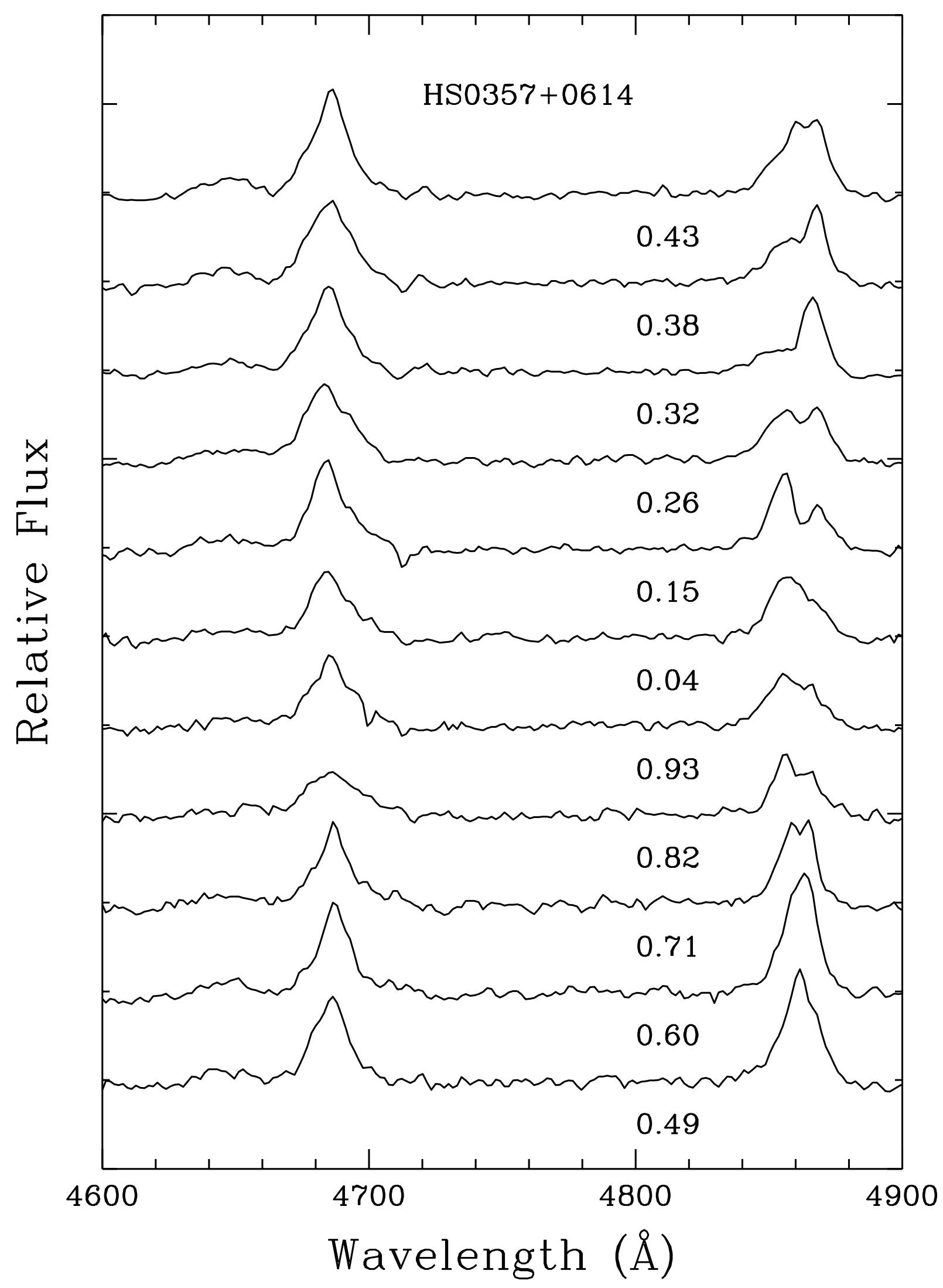




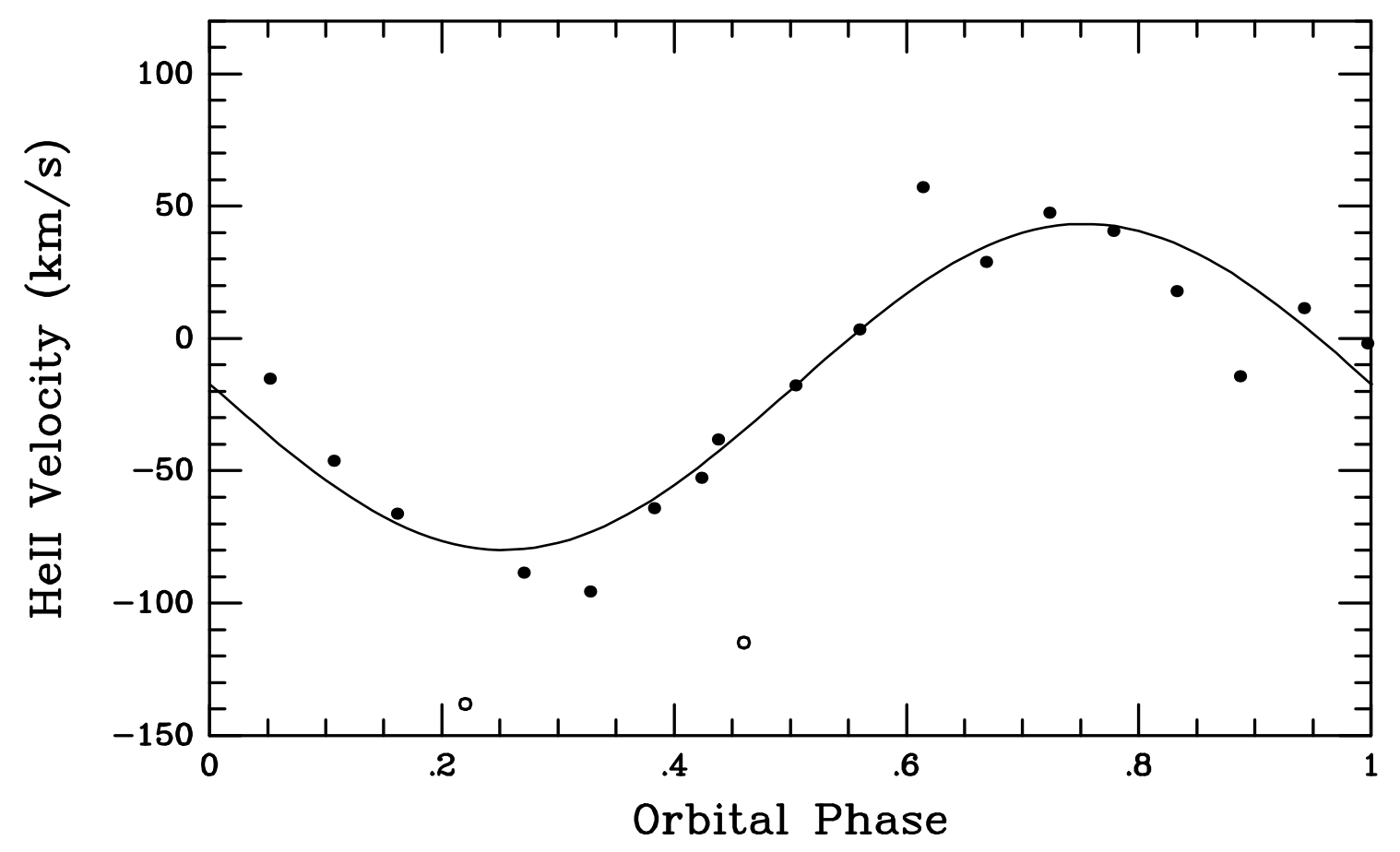



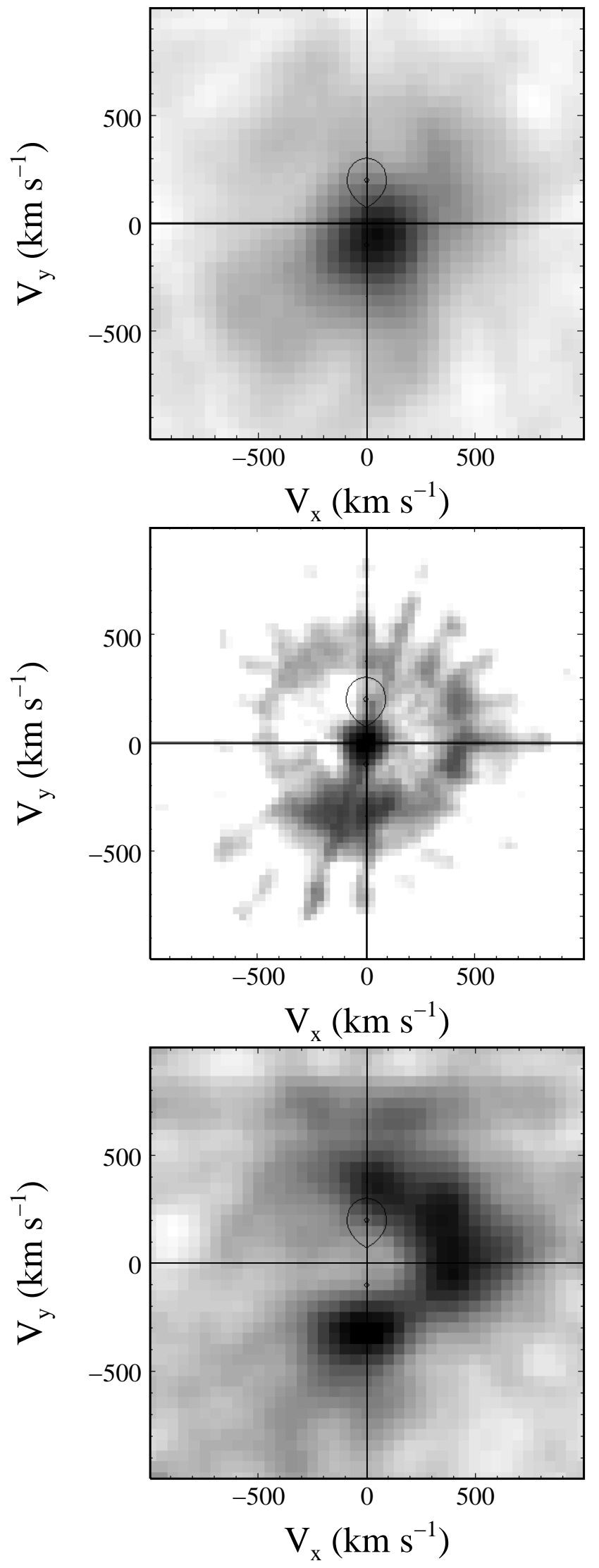